\title{
TWO CASES OF MYOCARDIAL INFARCTION IN CHILDREN
}

\author{
Hemant Narayan Ray ${ }^{1}$
}

\section{HOW TO CITE THIS ARTICLE:}

Hemant Narayan Ray. "Two Cases of Myocardial Infarction in Children". Journal of Evolution of Medical and Dental Sciences 2014; Vol. 3, Issue 56, October 27; Page: 12859-12863, DOI: 10.14260/jemds/2014/3709

ABSTRACT: Myocardial infarction in children is extremely rare and can have various etiologies. The following two case reports highlight rare but important causes of myocardial infarction in children.

KEYWORDS: Familial hypercholesterolemia, myocardial infarction, nephrotic syndrome, xanthomas.

INTRODUCTION: Myocardial infarction (MI) is very rare in children. It can have different etiologies. The common identified etiologies include Kawasaki disease, myocarditis, familial hypercholesterolemia, idiopathic or inherited cardiomyopathy, collagen vascular disease-induced coronary arteritis, substance abuse (cocaine, glue sniffing), trauma, complications of congenital heart disease surgery, - genetic disorders like progeria, pseudoxanthoma - elasticum, mucopolysaccharidoses etc., nephrotic syndrome, sepsis, occult malignancy and several other rare conditions.

We present two interesting cases of MI in children.

\section{CASE REPORTS:}

Case 1: A 12-year-old boy was diagnosed as nephrotic syndrome (minimal change disease) since the age of 7 years. He was on steroids for the last 4 years and had a satisfactory response. He presented with breathlessness, with episodes of paroxysmal nocturnal dyspnoea of 1 week duration. There was no chest pain. He was admitted to the emergency ward and treated for left ventricular failure (LVF).

He was also detected to have right hemiplegia with aphasia. The electrocardiogram showed typical ST elevation and pathological Q waves consistent with acute anteroseptal MI [Figure1]. A twoD echocardiogram revealed dilated left atrium and left ventricle (LV). There was severe LV systolic dysfunction with hypokinesia of the left anterior descending (LAD) territory. Troponin-T test was positive. Creatinine kinase-MB was in the normal range. A computed tomography (CT) scan of the brain showed recent infarct in the left fronto-temporo-parietal lobe.

Catheterization studies showed no evidence of any significant atherosclerotic coronary artery disease [Figure2]. The carotid arteries on both the sides were normal. Other investigations were: lipid profile - total cholesterol, $195 \mathrm{mg} / \mathrm{dl}$, low-density lipoprotein (LDL) cholesterol, $118 \mathrm{mg} / \mathrm{dl}$, triglycerides (TG), $191 \mathrm{mg} / \mathrm{dl}$, high-density lipoprotein (HDL) cholesterol, $38 \mathrm{mg} / \mathrm{dl}$, very low density lipoprotein (VLDL) cholesterol, $38 \mathrm{mg} / \mathrm{dl}$, significant proteinuria (albuminuria), serum hypoalbuminemia and thrombocytosis (platelet count, 9.3 lakhs $/ \mathrm{ml}$ ).

The serum homocysteine, rheumatoid factor IgM, anti-nuclear antibody, anti-double-stranded DNA and anti-cardiolipin-IgM and IgG levels were in the normal range. His heart failure and hemiparesis improved over 1 week on conservative management with diuretics, antiplatelets (aspirin and clopidogrel), nitrates, statins and low-molecular weight heparin. He was discharged in a stable hemodynamic condition. 


\section{CASE REPORT}

Figure 1: Electrocardiogram showing extensive anterior wall myocardial infarction (evolved).

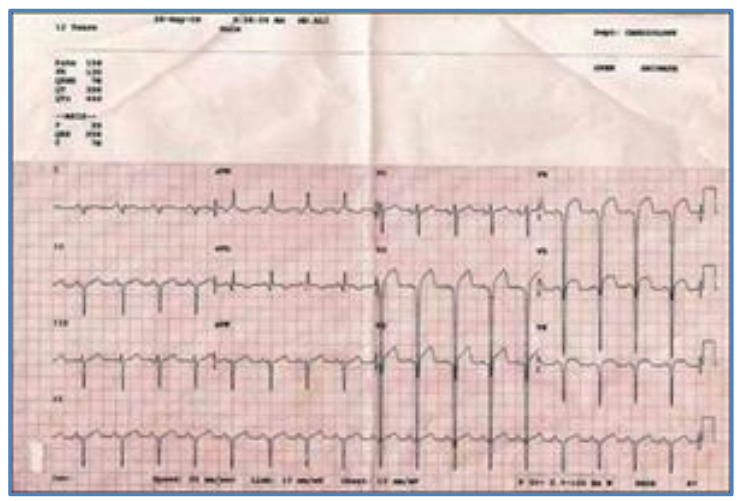

\section{Fig. 1}

Figure 2: Left coronary angiogram showing no evidence of significant coronary artery disease.

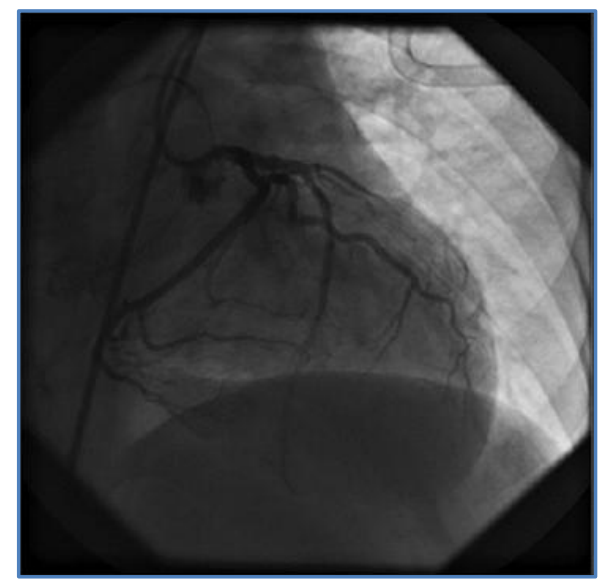

\section{Fig. 2}

Case 2: A 16-year-old boy presented with breathlessness and palpitations on minimal exertion for the past 1 month. He had no fever, respiratory symptoms or chest pain. He was a nonsmoker, nonhypertensive and non-diabetic. There was a family history of hypercholesterolemia in the younger sister. She was asymptomatic and was already on statin therapy. His younger brother died suddenly a year earlier. His parents were asymptomatic and their physical examination was normal. Unfortunately, we do not have their lipid profile.

The patient's physical examination revealed tendinous and tuberous xanthomas [Figures 3 \& 4], stria palmaris and arcus juveniles. Cardiovascular examination revealed mild cardiomegaly. The electrocardiogram showed poor progression of ' $R$ ' wave in lead V1 to V4. The chest $x$-ray showed cardiomegaly and there was evidence of pulmonary venous hypertension. The 2-D echocardiogram showed dilated LV, with severe LV systolic dysfunction and akinetic interventricular septum, moderate mitral regurgitation and moderate pulmonary artery hypertension. His lipid profile was as follows: total cholesterol, $440 \mathrm{mg} / \mathrm{dl}$, LDL cholesterol, $398 \mathrm{mg} / \mathrm{dl}$, HDL cholesterol, $30 \mathrm{mg} / \mathrm{dl}, \mathrm{TG}, 60$ $\mathrm{mg} / \mathrm{dl}$ and VLDL cholesterol, $12 \mathrm{mg} / \mathrm{dl}$. 


\section{CASE REPORT}

Figure 3: Photograph showing tendinous xanthomas on the elbow.

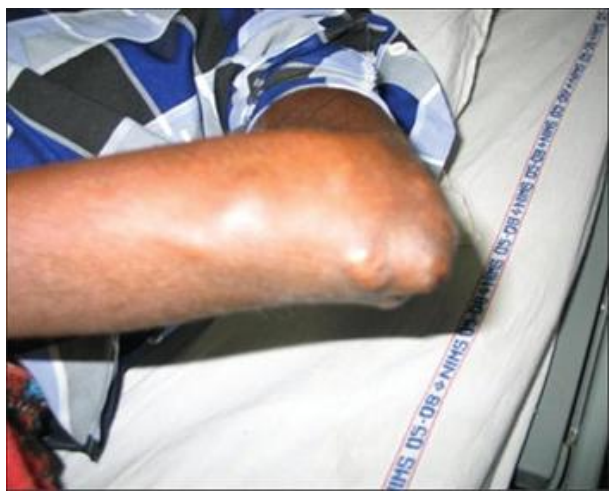

Fig. 3

Figure 4: Photograph showing tuberous xanthomas on the buttocks.

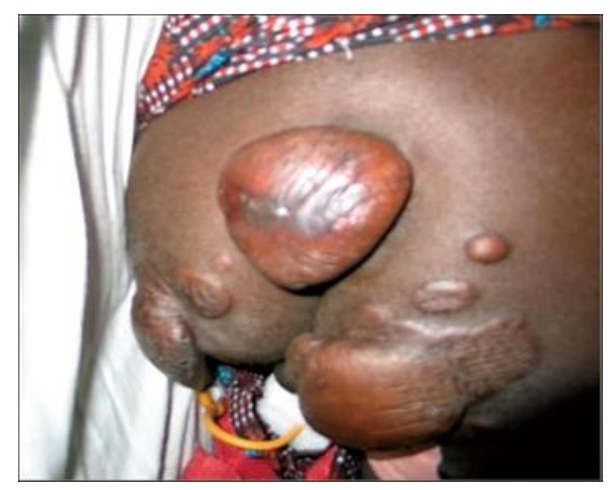

\section{Fig. 4}

His coronary angiogram showed $90 \%$ discrete, osteal stenosis of the left main coronary artery and severe proximal LAD disease with mid total occlusion - [Figure 5].

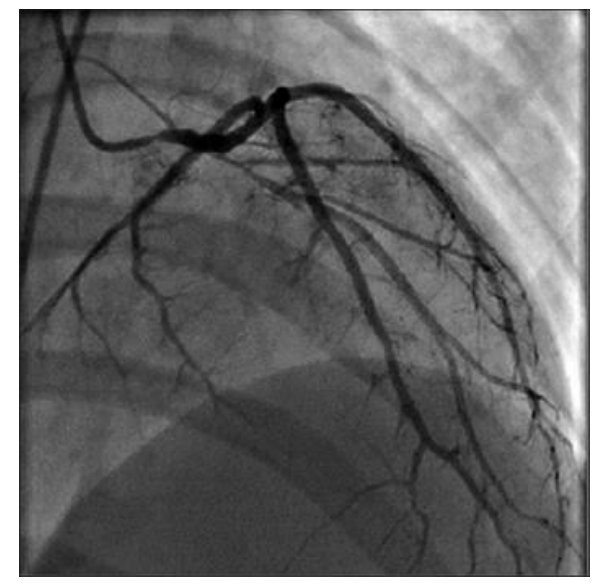

\section{Fig. 5}


Left coronary angiogram showing left main coronary artery-osteal $90 \%$ discrete stenosis and left anterior descending-proximal 90\% discrete stenosis and mid total occlusion.

He underwent coronary artery bypass grafting (CABG) on the next day uneventfully and he was discharged after 2 weeks on the following drugs: diuretics, ACE inhibitors, beta-blockers, antiplatelets (aspirin and clopidogrel) and statins (rosuvastatin and fenofibrate). He was asymptomatic during the $1^{\text {st }}$ month follow-up visit.

DISCUSSION: Our first case suggests nephrotic syndrome as a possible cause of thrombotic occlusion of the coronary and cerebral arteries, causing MI and cerebrovascular accident in this child. Ischemic heart disease is a rare complication of nephrotic syndrome. Only few cases are reported worldwide. Silva et al.[1] reported a 7-year-old boy with a 4-year history of steroid-unresponsive nephrotic syndrome due to mesangial proliferation disease and he presented with anteroseptal MI. However, cardiac catheterization showed no evidence of atherosclerotic coronary artery disease. Thrombotic occlusion of the left anterior descending artery was the likely cause of the event, similar to our patient. Hoppet al.[2] reported a 7-year-old boy with a 5-year history of steroid-unresponsive nephrotic syndrome due to minimal change disease presenting with acute MI.

The second case had familial hypercholesterolemia. It was clinically suspected because of tendinous and tuberous xanthomas with a strong family history. The lipid profile was consistent with Frederickson's Type IIa hypercholesterolemia (homozygous). However, detailed electrophoresis or genetic workup was not available.

MI in children with angiographically "normal" coronary arteries could occur because of hypercoagulable states - nephrotic syndrome and antiphospholipid syndrome (Hughes' syndrome), ${ }^{[3-6]}$ coronary artery spasm, ${ }^{[7-8]}$ coronary embolization ${ }^{[9]}$ and myocardial bridging.

Accelerated atherosclerosis, spontaneous dissections and aneurysms, ectasia and anomalous origin of coronary arteries may also rarely cause MI in children.

There are no controlled trials to guide early treatment of MI in this age group.[10] In conclusion, MI in children may result from diverse causes requiring different management approaches.

\section{REFERENCES:}

1. Silva JM, Oliveira EA, Marino VS, Oliveira JS, Torres RM, Ribeiro AL, et al. Premature acute myocardial infarction in a child with nephrotic syndrome. Pediatr Nephrol. 2002;17:169-72.

2. Hopp L, Gilboa N, Kurland G, Weichler N, Orchard TJ. Acute myocardial infarction in a young boy with nephrotic syndrome: A case report and review of literature. Pediatr Nephrol. 1994; 8: 290-4.

3. Fujimura 0, Gulamhusein S. Acute myocardial infarction: Thrombotic complications of nephrotic syndrome. Can J Cardiol. 1987; 3: 267-9.

4. Hamsten A, Norberg R, Bjorkholm M, de Faire U, Holm G. Antibodies to cardiolipin in young survivors of myocardial infarction: an association with recurrent cardio-vascular events. Lancet.1986;1: 113-6.

5. Manzar KJ, Padder FA, Conrad AR, Freeman I, Jonas EA. Acute myocardial infarction with normal coronary artery: A case report and review of literature. Am J Med Sci. 1997; 314: 342-5. 
6. Penny WJ, Colvin BT, Brooks N. Myocardial infarction with normal coronary arteries and factor XII deficiency. Br Heart J. 1985; 53: 230-4.

7. Simpsons RW, Edwards WD. Pathogenesis of cocaine induced ischemic heart disease-autopsy findings in a 21-year old man. Arch Pathol Lab Med. 1986; 110: 479-84.

8. Ross GS, Bell J. Myocardial infarction associated with inappropriate use of cocaine for treating epistaxis. Am J Emerg Med. 1992; 10: 219-222.

9. Agirbasli MA, Hansen DE, Byrd BF. Resolution of vegetations with anticoagulation after myocardial infarction in primary antiphospholipid syndrome. J Am Soc Echocardiogr. 1997; 10: 877-80.

10. Osula S, Bell GM, Hornung RS. Acute myocardial infarction in young adults: Cause and management. Postgrad Med J. 2002; 78: 27-30.

\section{AUTHORS:}

1. Hemant Narayan Ray

\section{PARTICULARS OF CONTRIBUTORS:}

1. Associate Professor, Department of Cardiology, Rajendra Institute of Medical Sciences.

\section{NAME ADDRESS EMAIL ID OF THE CORRESPONDING AUTHOR:}

Dr. Hemant Narayan Ray, Department of Cardiology, Rajendra Institute of Medical Sciences, Bariatu Road, Ranchi-834009, Jharkhan, India.

Email: hemantnarayanray@yahoo.com

Date of Submission: 12/09/2014. Date of Peer Review: 13/09/2014. Date of Acceptance: 22/10/2014. Date of Publishing: 27/10/2014. 